\title{
Emission Spectroscopy of OH Radical in Water-Argon Arc Plasma Jet
}

\begin{abstract}
Alan Mašláni and Viktor Sember
Institute of Plasma Physics v.v.i., Za Slovankou 3, 18200 Prague, Czech Republic

Correspondence should be addressed to Alan Mašláni; maslani@ipp.cas.cz

Received 10 January 2014; Revised 15 March 2014; Accepted 17 March 2014; Published 7 April 2014

Academic Editor: Manuel A. Gonzalez

Copyright (C) 2014 A. Mašláni and V. Sember. This is an open access article distributed under the Creative Commons Attribution License, which permits unrestricted use, distribution, and reproduction in any medium, provided the original work is properly cited.

Emission spectra of $\mathrm{OH}$ radical are studied in the plasma jet generated by a plasma torch with hybrid water-argon stabilization. Plasma jet is located in a chamber with pressures $4 \mathrm{kPa}$ and $10 \mathrm{kPa}$. In spite of high temperatures of produced plasma, $\mathrm{OH}$ spectra are observed in a large area of the jet. $\mathrm{OH}$ spectra are used to obtain rotational temperatures from the Boltzmann plots of resolved rotational lines. Due to line-of-sight integration of radiation, interpretation of the temperatures is not straightforward. It seems that excited $\mathrm{OH}$ molecules can be formed by various mechanisms, mainly in the outer parts of the jet, where thermal processes are not as dominant as in the hot central region.
\end{abstract}

\section{Introduction}

Emission spectra of $\mathrm{OH}$ molecules are frequently observed in different types of plasmas, usually when water is present as one of the initial species. Even in cases of relatively low amount of water in plasma forming medium, for instance, air $[1,2]$, OH spectra can be distinguished and exploited for further processing. It is thus natural that $\mathrm{OH}$ spectra are often studied, although $\mathrm{OH}$ radical is only one of the components, which create the chemistry of plasmas. However, understanding of $\mathrm{OH}$ production and measurement of its temperature and density is important also from the point of view of its application as a reactive radical which is formed in laboratory and industrial plasmas [3-6] and flames [7, 8]. Moreover, $\mathrm{OH}$ radical is found also in Earth atmosphere where it plays important role in different chemical reactions and is an object of many studies [9-11]. It is evident that $\mathrm{OH}$ emission is observed from many plasma environments over a wide range of pressures and temperatures. On the other hand, for the high power arc plasmas and plasma torches, $\mathrm{OH}$ molecules are investigated quite seldom [12, 13]. It is partly caused by the fact that plasma torches often work with plasma forming gases, which do not allow formation of $\mathrm{OH}$ in relevant amount and their emission spectra contain strong lines of other species. Another reason is that for these kinds of plasma sources emphasis is given in investigation of central regions of the arc columns where atomic and ionic species dominate.

A plasma torch, in which water or mixture of water and argon is used to stabilize the arc discharge [14], is a natural source of $\mathrm{OH}$ radicals. Some $\mathrm{OH}$ spectroscopic measurements were already performed on it by our group [13]; comparison between $\mathrm{OH}$ rotational temperature and argon excitation and ionization temperatures was presented. $\mathrm{OH}$ is probably involved in two main applications of this torch, plasma spraying [15] and plasma aided gasification of waste materials [16]. In the latter case, for example, $\mathrm{OH}$ is an important radical in the complicated chemistry, leading to the formation of syngas, that is, mixture of $\mathrm{CO}$ and $\mathrm{H}_{2}$.

It is generally supposed that $\mathrm{OH}$ in plasmas is produced mainly by dissociation of water molecules, which is supported experimentally and theoretically [6]. On the other hand, it is usually difficult to evaluate which of reaction channels is dominant in particular conditions. However, in case of mentioned plasma torch with water as plasma forming medium, applied arc current is in order of hundreds of amperes and arc power reaches tens of kilowatts. Then, in the arc channel with diameter of few millimetres, water 


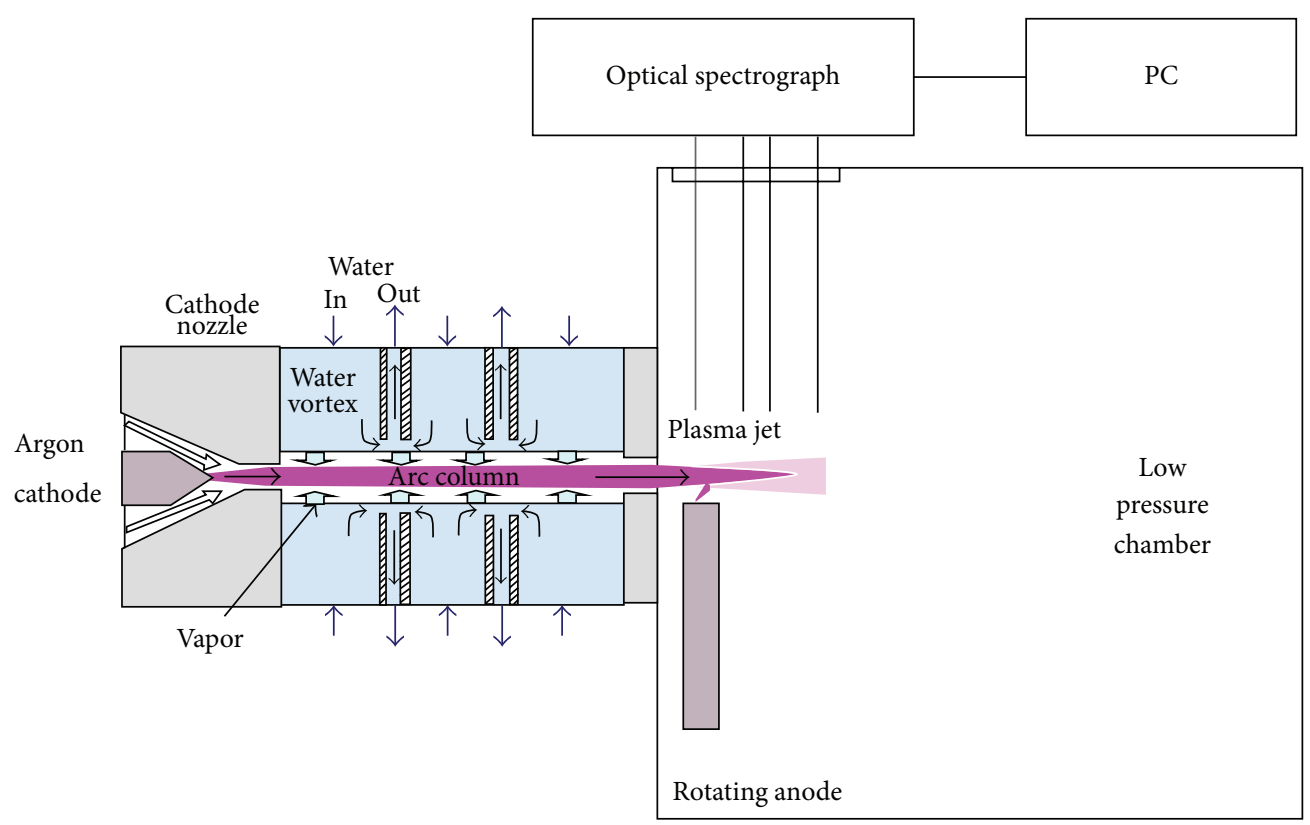

FIGURE 1: Schematic view of the plasma torch attached to the low pressure chamber and plasma jet observed by the spectrograph.

is probably completely dissociated and temperatures can exceed $20000 \mathrm{~K}$, depending on particular conditions. As a result, one can assume that in the recombining jet some $\mathrm{OH}$ molecules can be formed by association of oxygen and hydrogen, for instance, or by other mechanisms without presence of water molecule. However, we must still take into account mechanisms involving water molecules, which are present in the colder regions around plasma column. For example, charge transfer between argon ion and water molecule forms $\mathrm{H}_{2} \mathrm{O}^{+}$, from which $\mathrm{OH}$ in excited state is formed by dissociative recombination.

In this work, above-mentioned plasma torch with waterargon stabilization is used as a source of plasma. Compared with plasma jets in gases without water, the jet with water is characterized by high enthalpy $(\sim 200 \mathrm{MJ} / \mathrm{kg})$, temperature $(\sim 20000 \mathrm{~K})$, and velocity $(\sim 6000 \mathrm{~m} / \mathrm{s})$; more details and comparison of different torches can be found elsewhere [14, 17]. The jet is located in a low pressure environment, which simplifies $\mathrm{OH}$ observation by optical emission spectroscopy. Moreover, in these conditions the $\mathrm{OH}$ spectra are observable in large part of the jet, in the direction of the hot core of the arc column, and in the outer parts of the free jet where visible radiation is quite weak. In comparison with our previous paper [13], in this work spectra with higher resolution are studied; thus Boltzmann plots using individual rotational transitions can be constructed. Also the mapping of the plasma jet is more detailed including two different pressures. As a result, we get better insight into the distribution of $\mathrm{OH}$ and its formation in this plasma.

\section{Experimental Setup}

Schematic view of the experiment is shown in Figure 1. The arc is stabilized by the argon in the cathode region and by the water vortex surrounding substantial part of the arc column. In the present experiment, the arc current is $250 \mathrm{~A}$ (voltage is about $200 \mathrm{~V}$ and power is about $50 \mathrm{~kW}$ ) and the argon flow rate is $12 \operatorname{sim}\left(5 \times 10^{21}\right.$ particles per second). Water supply system contains high amount of water, from which only small part evaporates to plasma. It is thus difficult to determine how much water goes to the arc; estimations (based also on the arc modelling) give values of water evaporation rate about $0.3 \mathrm{~g} / \mathrm{s}\left(1 \times 10^{22}\right.$ particles per second $)$. This value, however, can have quite high error. Disc-shaped anode with thickness $20 \mathrm{~mm}$, manufactured from copper, is rotating with high velocity in order to assure uniform erosion and is cooled by water. The anode is located outside of the arc chamber $2 \mathrm{~mm}$ from the nozzle exit in horizontal direction. The length of the arc column inside the torch body, that is, distance from the cathode to nozzle, is $56 \mathrm{~mm}$. Diameter of the arc column inside the torch is approximately $6 \mathrm{~mm}$; it is defined by water wall surrounding the arc. The nozzle connecting arc chamber with outer environment has diameter of $5 \mathrm{~mm}$. From this description and from Figure 1, it is evident that we are able to observe not only free expanding jet but also part of the arc between nozzle and anode. The torch is attached to the chamber evacuated by a rotary vacuum pump which allows decreasing the pressure from atmospheric pressure down to $1 \mathrm{kPa}$.

Image of the plasma jet is projected through the lens with focal distance $300 \mathrm{~mm}$ onto the entrance of the optical cable, which transfers signal to the monochromator Jobin Yvon-Spex Triax 550. The optical cable consists of 41 fibers arranged in the line, resembling the entrance slit of the monochromator (Figure 2). The height of the line is $4 \mathrm{~mm}$; with magnification 0.36 given by lens, we have the height of the measured region about $11 \mathrm{~mm}$. The output spectrum is detected by the iCCD detector with $1024 \times 256$ pixels 


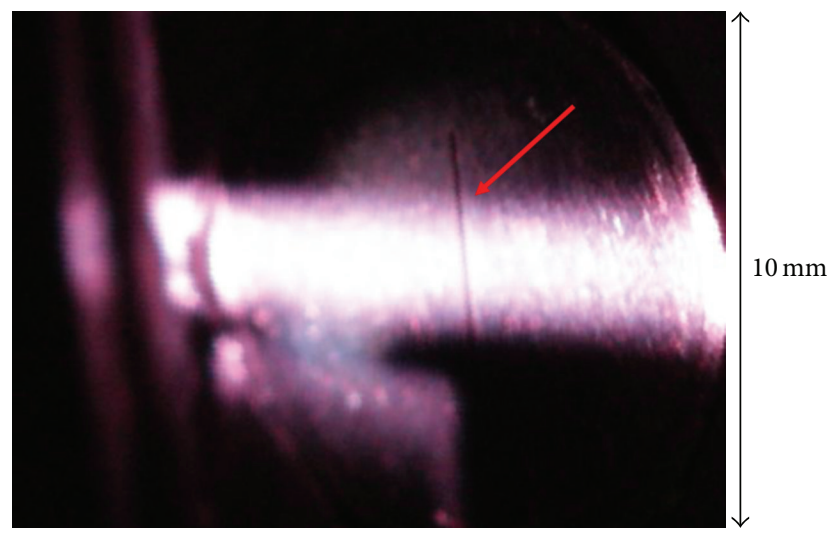

FIgURE 2: Photograph of the entrance of the optical cable with the image of the plasma jet. Red arrow shows the entrance of the optical fibre system with height of $4 \mathrm{~mm}$.

connected to the CCD 3000 controller and to the PC. Measured spectra of $\mathrm{OH}$ are calibrated using deuterium lamp which is suitable as calibration standard in the spectral region 250-350 $\mathrm{nm}$. All measurements are time averaged in the scale from hundreds of milliseconds to seconds.

\section{OH Emission Spectra}

The spectra of $\mathrm{OH}$ electronic transition $\mathrm{A}^{2} \Sigma \rightarrow \mathrm{X}^{2} \Pi$ with the bandhead at $306.4 \mathrm{~nm}$ were measured using the grating with 3600 grooves $/ \mathrm{mm}$, which gives resolution of $0.019 \mathrm{~nm}$ FWHM. With this relatively high resolution, the individual rotational lines can be studied. Examples of typical spectra are shown in Figure 3. Two spectral windows with central wavelengths $310 \mathrm{~nm}$ and $316 \mathrm{~nm}$ were used, in order to cover main part of the spectrum.

Since many of the rotational lines are resolved, the method of Boltzmann plot is used to obtain rotational temperature instead of comparison of experimental and simulated spectra. Two spectral windows allow using resolved lines of $\mathrm{Q}_{1}$ branch up to rotational quantum number $J=24$.

It should be noted that $\mathrm{OH}$ is the only molecule, which is possible to observe in emission spectrum from this plasma in spectral range $200-1000 \mathrm{~nm}$. Except it, we are able to detect only lines of atomic argon, oxygen, and hydrogen and also singly ionized argon and oxygen. In spite of the fact that the plasma jet is surrounded by air, we do not see any molecules containing nitrogen, as $\mathrm{NH}, \mathrm{N}_{2}, \mathrm{~N}_{2}^{+}$, or NO. It is caused partly by the low pressure environment. As for $\mathrm{N}_{2}$ and $\mathrm{N}_{2}{ }^{+}$ excited states, it was reported that they are more likely to be present in dry air plasma, probably because of their fast decomposition in the reactive environment of water plasma [3]. In our plasma, the mechanism can be similar to the above-mentioned decomposition of water by charge transfer and dissociative recombination, which produces excited $\mathrm{OH}$. Also for nitrogen and oxygen molecules, charge transfer with argon ion may produce $\mathrm{N}_{2}^{+}$and $\mathrm{O}_{2}{ }^{+}$ions, which are then immediately dissociated. However, the product of this dissociation is not molecule as in case of water but excited atomic nitrogen and oxygen. Anyway, the complete

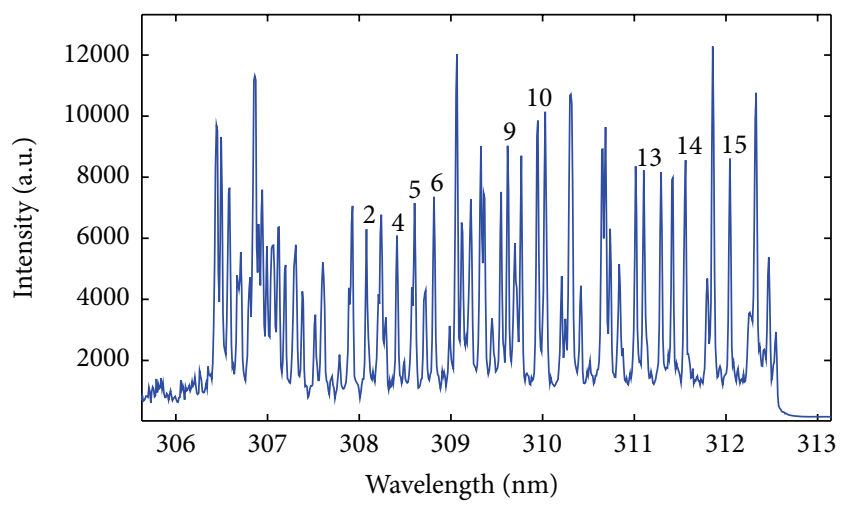

(a)

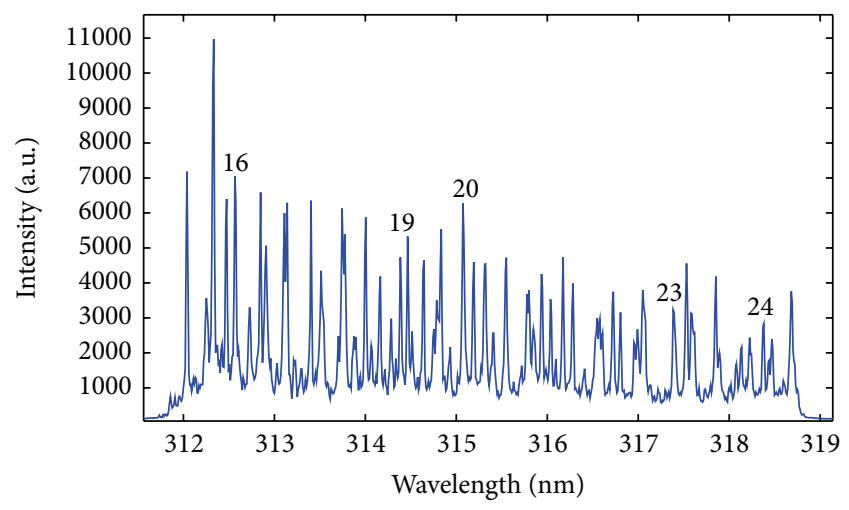

(b)

FIgURE 3: Typical spectra of $\mathrm{OH}$ with the central wavelengths $310 \mathrm{~nm}$ (a) and $316 \mathrm{~nm}$ (b). Lines of $\mathrm{Q}_{1}$ branch used for rotational temperature evaluation are denoted by their rotational quantum number $J$.

chemistry of this mixture is for sure more complicated than emission spectra reveal.

It was mentioned already that $\mathrm{OH}$ spectra can be measured almost in any position of the plasma jet, from the hot arc column between nozzle and anode to cold outer parts of the jet. It means, however, that resulting spectrum is probably composed of contributions from the regions with substantially different conditions. It seems that mechanism of charge transfer and dissociative recombination can produce relevant population of excited $\mathrm{OH}$ in the outer parts of the jet. However, radial profile of $\mathrm{OH}$ intensity still has maximum in the jet axis. Anyway, as a result, $\mathrm{OH}$ radiation distribution in relatively thick column is complicated and can be hardly approximated by cylindrical symmetry. Therefore, Abel inversion, which is commonly performed for atomic and ionic species, cannot be done with adequate precision and thus all results in this work are obtained from line of sight integrated values.

\section{Results and Discussion}

Rotational temperatures were calculated from the measured spectra in the large area of the jet, from the nozzle (axial distance of $0 \mathrm{~mm}$ ) up to $70 \mathrm{~mm}$ in the axial direction and 
$5 \mathrm{~mm}$ from the jet axis in the radial direction. The radial range is given by the geometry of the spectroscopic setup and by the size of the entrance of the optical cable, as was mentioned in Section 2. The jet axis (radial distance of $0 \mathrm{~mm}$ ) is defined as a position in radial profile where continuum and atomic or ionic lines reach maximum intensity. This point is not always the axis of the nozzle, as can be deduced also from the jet images in Figure 4. Measurements were done for two chamber pressures, $10 \mathrm{kPa}$ and $4 \mathrm{kPa}$. To imagine the plasma jet in visible light, the photographs for these pressures are included (Figure 4). For $10 \mathrm{kPa}$, the jet structure is more pronounced, showing clearly the expansion region at approximately $10 \mathrm{~mm}$ from the nozzle with lower radiation intensity, where the supersonic velocity and low density of plasma are present. On the other hand, for $4 \mathrm{kPa}$, the jet is more diffuse and supersonic structure is not apparent in visible light.

Figure 5 shows the maps of rotational temperatures in the jet for $10 \mathrm{kPa}$ and for $4 \mathrm{kPa}$. The resolution is higher for radial direction, where the distance between $-5 \mathrm{~mm}$ and $5 \mathrm{~mm}$ is covered by 166 pixels of CCD chip (the second dimension of the CCD represents spectral resolution). On the other hand, in the axial distance, the individual points of measurement correspond to manual movement of the optical fibre along the image of the jet. Hence, the resolution in this direction is lower; the distance from the nozzle up to $70 \mathrm{~mm}$ is covered by only 15 points.

It should be mentioned again that presented temperatures do not represent local values; measured spectra are integrated along the line of sight, since the symmetry of the measured profiles of $\mathrm{OH}$ is insufficient to perform Abel inversion. Thus, in fact, spectra are composed of $\mathrm{OH}$ emissions from various parts of plasma with different properties; this will be discussed also further. Moreover, in spite of low pressure, even self-absorption cannot be excluded, since the amount of $\mathrm{OH}$ might be high in relatively thick plasma column. These restrictions should be kept in mind before drawing any conclusions; however, at least some information can be obtained from presented results.

Both images in Figure 5 reveal minimum of temperature at about $10 \mathrm{~mm}$ from the nozzle. This corresponds to the expansion region observed in photograph in Figure 4 for $10 \mathrm{kPa}$. For $4 \mathrm{kPa}$, the local minimum in the radiation intensity in this region is not visible anymore; however, rotational temperature has minimum in this position similarly as for $10 \mathrm{kPa}$. For $10 \mathrm{kPa}$, one more relatively strong minimum of temperature is observed at $48 \mathrm{~mm}$, although optical image does not indicate it. It can show the position of one more expansion zone. For $4 \mathrm{kPa}$, the variations of temperature along the jet axis downstream the first minimum are smaller, which corresponds to diffuse and more uniform jet.

As for the radial profiles of temperatures, they have always minimum near the jet axis with slight increase towards the jet periphery. Relatively small differences in temperatures in different parts of the jet can be attributed to line-of-sight integration of spectra. Indeed, $\mathrm{OH}$ spectra have been measured in even higher radial and axial distances than presented here. Thus, when measuring spectrum in the direction of jet axis, contributions from the whole thickness of the plasma

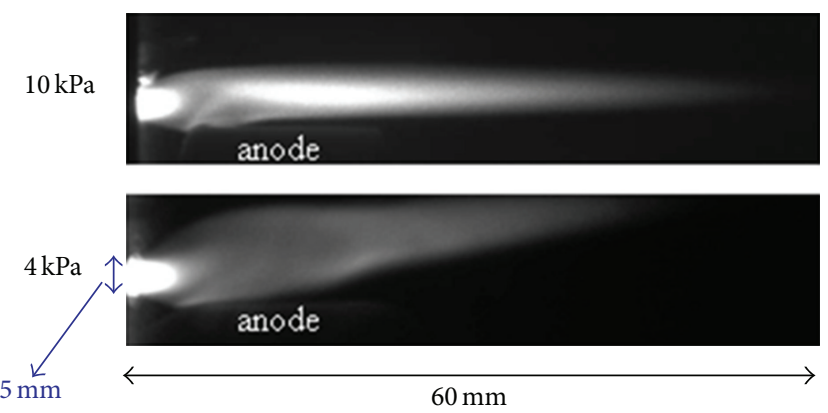

FIGURE 4: Photographs of the plasma jet in visible light captured by fast shutter camera for chamber pressures $10 \mathrm{kPa}$ and $4 \mathrm{kPa}$. Radial scale is marked using the diameter of the nozzle, which is $5 \mathrm{~mm}$.
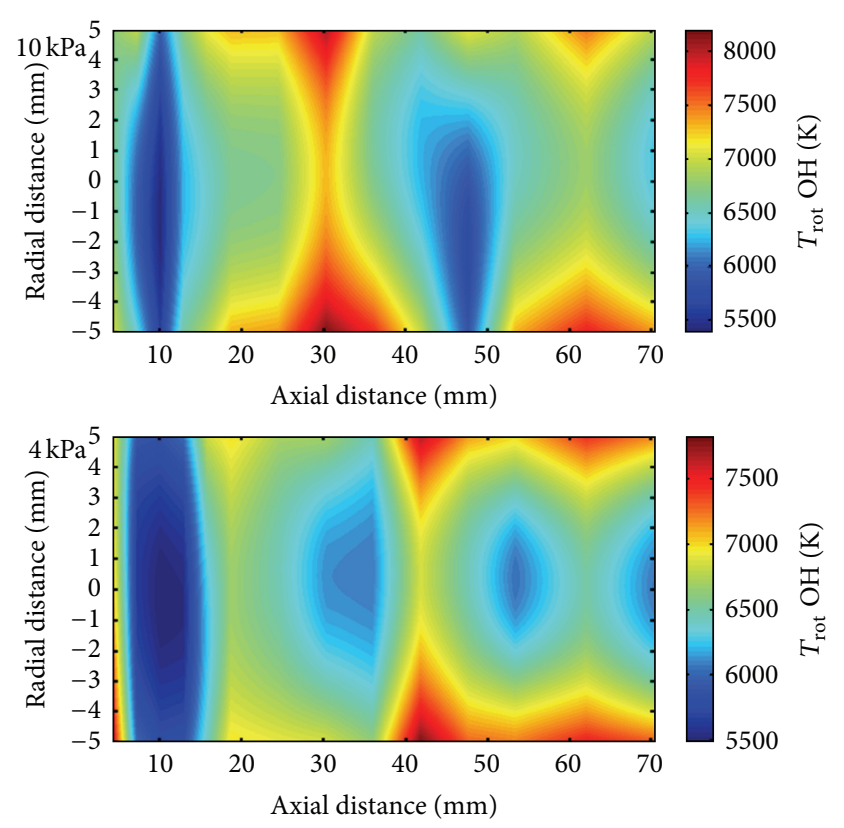

FIGURE 5: Rotational temperature distribution in the jet for chamber pressures $10 \mathrm{kPa}$ and $4 \mathrm{kPa}$.

column are summed. We should also keep in mind that hot core of the plasma next to the exit from the nozzle, which has temperature, according to local measurements of atomic and ionic lines, more than $10000 \mathrm{~K}$, is in principle completely dissociated and contains negligible amount of $\mathrm{OH}$. On the other hand, measurement in the periphery integrates more radiation from colder outer parts, where amount of $\mathrm{OH}$ should be higher. Anyway, as was mentioned already, resulting radial profile of $\mathrm{OH}$ radiation intensity has always maximum approximately in the jet axis.

Nevertheless, increase of temperature in the jet periphery is evident. We can try to understand it with the help of examples of Boltzmann plots from different radial positions; see Figure 6. In this figure, first, Boltzmann plot corresponds to the position on the jet axis. It is clear that temperature is well defined with good accuracy. Points, which do not fit the line perfectly, probably correspond to nonideally resolved lines rather than to some nonequilibrium effects. Figure 6(b) 


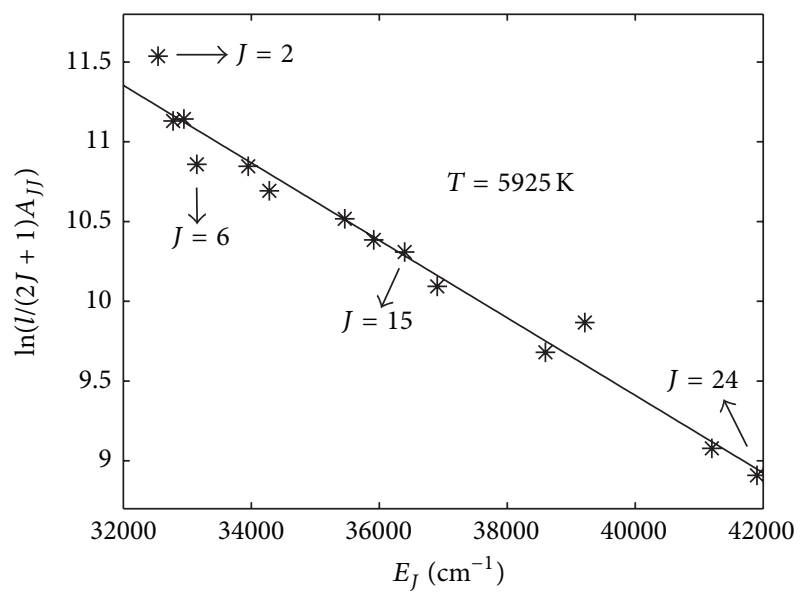

(a)

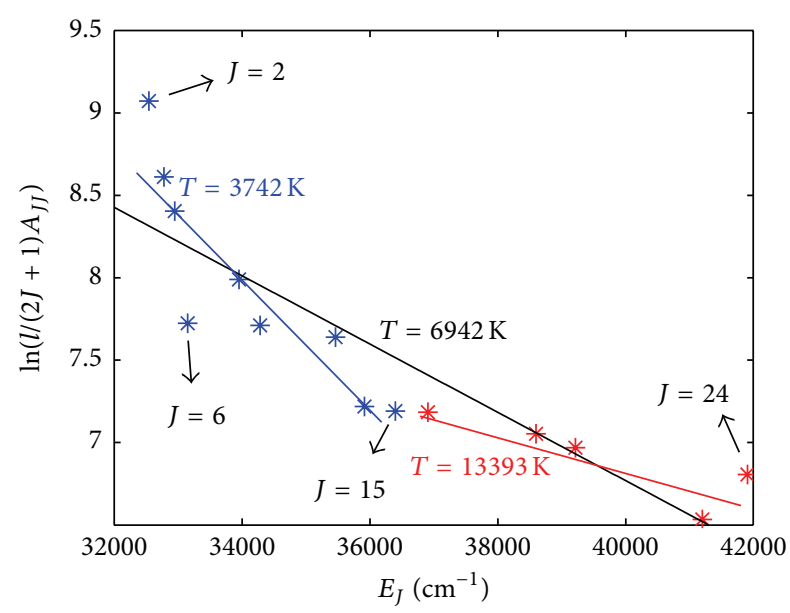

(b)

FIgURE 6: Typical Boltzmann plots in the jet axis (a) and in the jet periphery (b). Points correspond to lines marked in Figure 3 by rotational quantum numbers $J$; here, some $J$ values are also shown.

shows typical Boltzmann plot from the jet periphery. In this case, temperature is higher than in the axis $(T=$ $6942 \mathrm{~K}$ ), but also with higher statistical error. Moreover, it is possible to choose lines with low rotational number (up to approximately $J=16$ ) and a higher one (from $J=16$ to $J=$ $24)$, which define substantially different temperatures with lower error $(T=3742 \mathrm{~K}$ and $T=13393 \mathrm{~K})$. It means that higher temperature from all lines is caused by the higher population of higher rotational states with respect to lower ones. This effect is described in literature for low pressure and even for atmospheric pressure plasmas [18]; it has to do with different ways of $\mathrm{OH}$ formation. Application of this effect to arcjet plasmas is not necessarily straightforward; however, one can see at least indication of similar behaviour. We can suppose that in the jet periphery relevant amount of water molecules allows the mechanism of charge transfer and dissociative recombination to become important in formation of excited $\mathrm{OH}$.

As the atomic and ionic lines reliably show that axis temperatures next to the nozzle and anode are higher than
$10000 \mathrm{~K}$, presented rotational temperatures near the jet axis, in spite of well-defined Boltzmann plots, apparently do not represent kinetic temperatures of this hot plasma. These temperatures rather describe the region surrounding the hot core, where the plasma is still thermalized to establish Boltzmann distribution even for high rotational numbers and where at the same time there is already low enough temperature for considerable amount of $\mathrm{OH}$ molecules to exist. On the other hand, in the jet periphery where thermal equilibrium is probably violated, excited $\mathrm{OH}$ seems to be formed by other mechanisms (from water by charge transfer and dissociative recombination), which is favouring higher rotational states.

\section{Conclusion}

Plasma jet generated by plasma torch with water-argon stabilization was studied by emission spectroscopy. Thermal plasma originating from water naturally contains considerable amount of $\mathrm{OH}$ molecules. Measurement of its emission spectrum allows studying rotational population distribution of excited $\mathrm{OH}$ and calculating rotational temperature. The results indicate that $\mathrm{OH}$ production in recombining free jet is complicated process comprising various reactions and depending on exact position in the jet; therefore, obtained rotational temperature should be interpreted with care.

\section{Conflict of Interests}

The authors declare that there is no conflict of interests regarding the publication of this paper.

\section{Acknowledgment}

This work was supported by the Grant Agency of the Czech Republic under Project no. GAP205/11/2070.

\section{References}

[1] C. O. Laux, T. G. Spence, C. H. Kruger, and R. N. Zare, "Optical diagnostics of atmospheric pressure air plasmas," Plasma Sources Science and Technology, vol. 12, no. 2, pp. 125-138, 2003.

[2] Z. Machala, M. Janda, K. Hensel et al., "Emission spectroscopy of atmospheric pressure plasmas for bio-medical and environmental applications," Journal of Molecular Spectroscopy, vol. 243, no. 2, pp. 194-201, 2007.

[3] B. Benstaali, P. Boubert, B. G. Cheron, A. Addou, and J. L. Brisset, "Density and rotational temperature measurements of the $\mathrm{OH}$ and NO radicals produced by a gliding arc in humid air," Plasma Chemistry and Plasma Processing, vol. 22, no. 4, pp. 553-571, 2002.

[4] R. Ono and T. Oda, "Measurement of gas temperature and $\mathrm{OH}$ density in the afterglow of pulsed positive corona discharge," Journal of Physics D: Applied Physics, vol. 41, no. 3, Article ID 035204, 2008.

[5] N. Srivastava, C. Wang, and T. S. Dibble, "A study of OH radicals in an atmospheric AC discharge plasma using near infrared 
diode laser cavity ringdown spectroscopy combined with optical emission spectroscopy," European Physical Journal D, vol. 54, pp. 77-86, 2009.

[6] P. Bruggeman and D. C. Schram, "On OH production in water containing atmospheric pressure plasmas," Plasma Sources Science and Technology, vol. 19, Article ID 045025, 2010.

[7] K. Nyholm, "Measurements of $\mathrm{OH}$ rotational temperatures in flames by using polarization spectroscopy," Optics Communications, vol. 111, no. 1-2, pp. 66-70, 1994.

[8] A. T. Hartlieb, B. Atakan, and K. Kohse-Höinghaus, "Temperature measurement in fuel-rich non-sooting low-pressure hydrocarbon flames," Applied Physics B: Lasers and Optics, vol. 70, no. 3, pp. 435-445, 2000.

[9] F. Sigernes, N. Shumilov, C. S. Deehr, K. P. Nielsen, T. Svenøe, and O. Havnes, "Hydroxyl rotational temperature record from the auroral station in Adventdalen, Svalbard $\left(78^{\circ} \mathrm{N}, 15^{\circ} \mathrm{E}\right)$, Journal of Geophysical Research A: Space Physics, vol. 108, no. 9, article 1342, 2003.

[10] R. L. Mauldin III, S. Madronich, S. J. Flocke, F. L. Eisele, G. J. Frost, and A. S. H. Prevot, "New insights on OH: measurements around and in clouds," Geophysical Research Letters, vol. 24, no. 23, pp. 3033-3036, 1997.

[11] M. H. Stevens, R. L. Gattinger, J. Gumbel et al., "First UV satellite observations of mesospheric water vapor," Journal of Geophysical Research D: Atmospheres, vol. 113, no. 12, Article ID D12304, 2008.

[12] T. Watanabe and Narengerile, "Role of $\mathrm{CH}, \mathrm{CH}_{3}$ and $\mathrm{OH}$ radicals in organic compound decomposition by water plasmas," in Proceedings of the 20th International Symposium on Plasma Chemistry, Philadelphia, Pa, USA, July 2011, http://www.ispc-conference.org/ispcproc/ispc20/148.pdf.

[13] A. Mašláni and V. Sember, "Rotational distribution of $\mathrm{OH}$ molecules in supersonic thermal plasma jet," High Temperature Material Processes, vol. 13, no. 2, pp. 205-215, 2009.

[14] M. Hrabovský, "Generation of thermal plasmas in liquidstabilized and hybrid dc-arc torches," Pure and Applied Chemistry, vol. 74, no. 3, pp. 429-433, 2002.

[15] T. Kavka, J. Matějíček, P. Ctibor, A. Mašláni, and M. Hrabovský, "Plasma spraying of copper by hybrid water-gas DC arc plasma torch," Journal of Thermal Spray Technology, vol. 20, no. 4, pp. 760-774, 2011.

[16] M. Hrabovský, "Plasma aided gasification of biomass, organic waste and plastics," in Proceedings of the 30th International Conference on Phenomena in Ionized Gases (ICPIG '11), Belfast, Northern Ireland, 2011, http://mpserver.pst.qub.ac.uk/sites/ 406_GEN_Hrabovsky.pdf.

[17] M. Hrabovsky, V. Kopecký, V. Sember, T. Kavka, O. Chumak, and M. Konrad, "Properties of hybrid water/gas dc arc plasma torch," IEEE Transactions on Plasma Science, vol. 34, no. 4, pp. 1566-1575, 2006.

[18] P. Bruggeman, D. C. Schram, M. G. Kong, and C. Leys, "Is the rotational temperature of $\mathrm{OH}(\mathrm{A}-\mathrm{X})$ for discharges in and in contact with liquids a good diagnostic for determining the gas temperature?" Plasma Processes and Polymers, vol. 6, no. 11, pp. 751-762, 2009. 

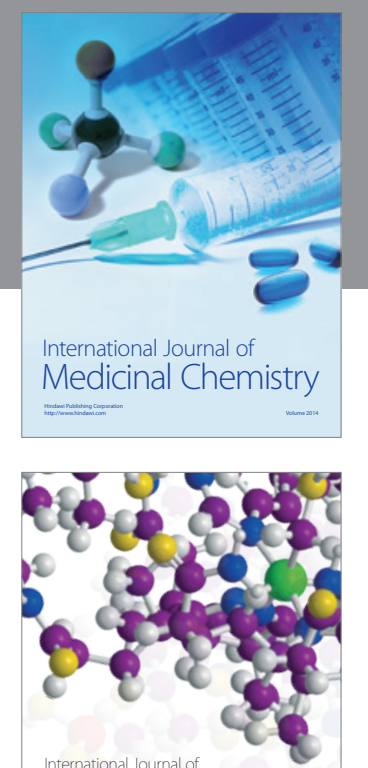

\section{Carbohydrate} Chemistry

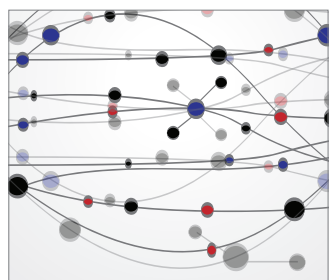

The Scientific World Journal
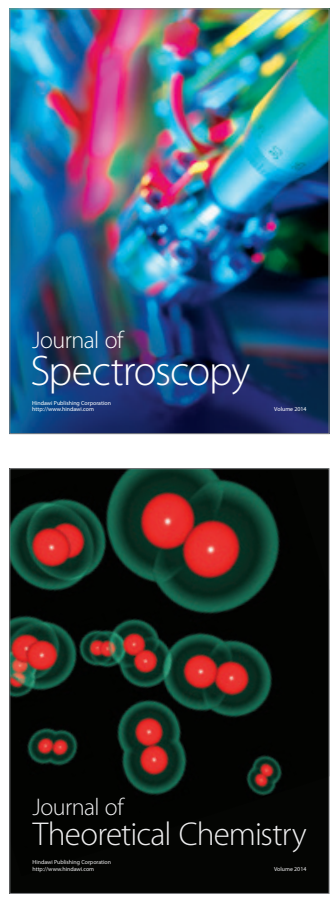
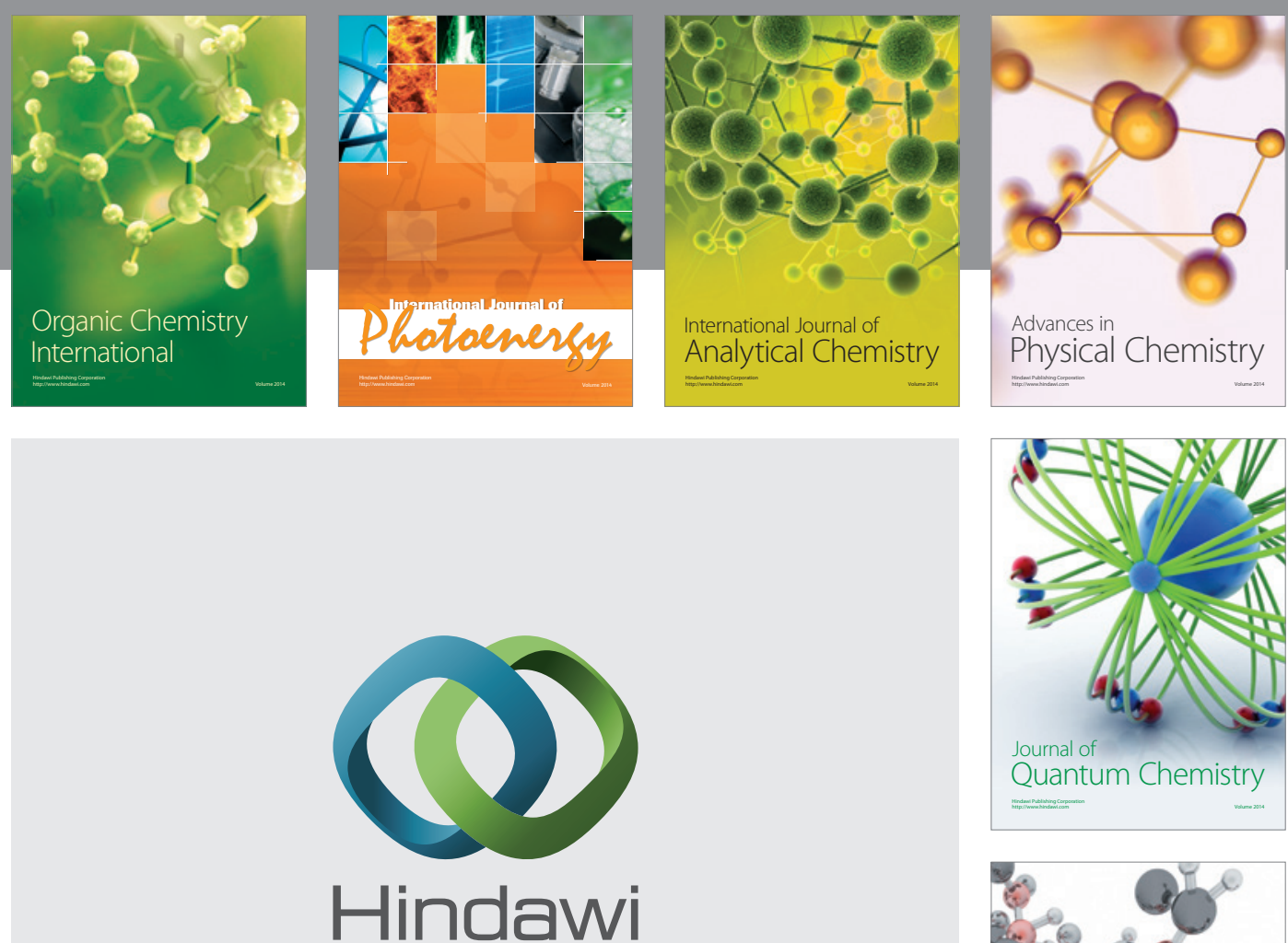

Submit your manuscripts at

http://www.hindawi.com

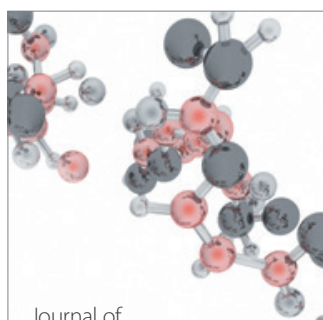

Analytical Methods

in Chemistry

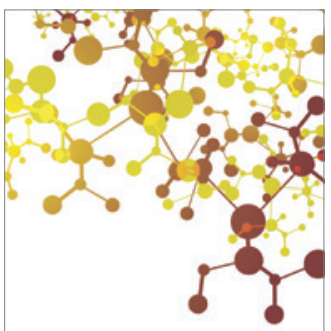

Journal of

Applied Chemistry

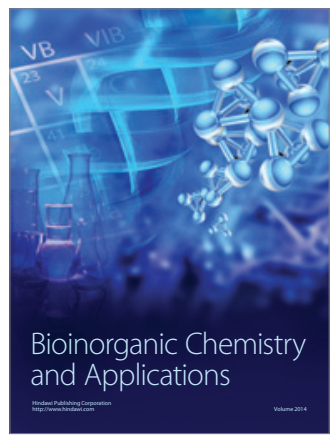

Inorganic Chemistry
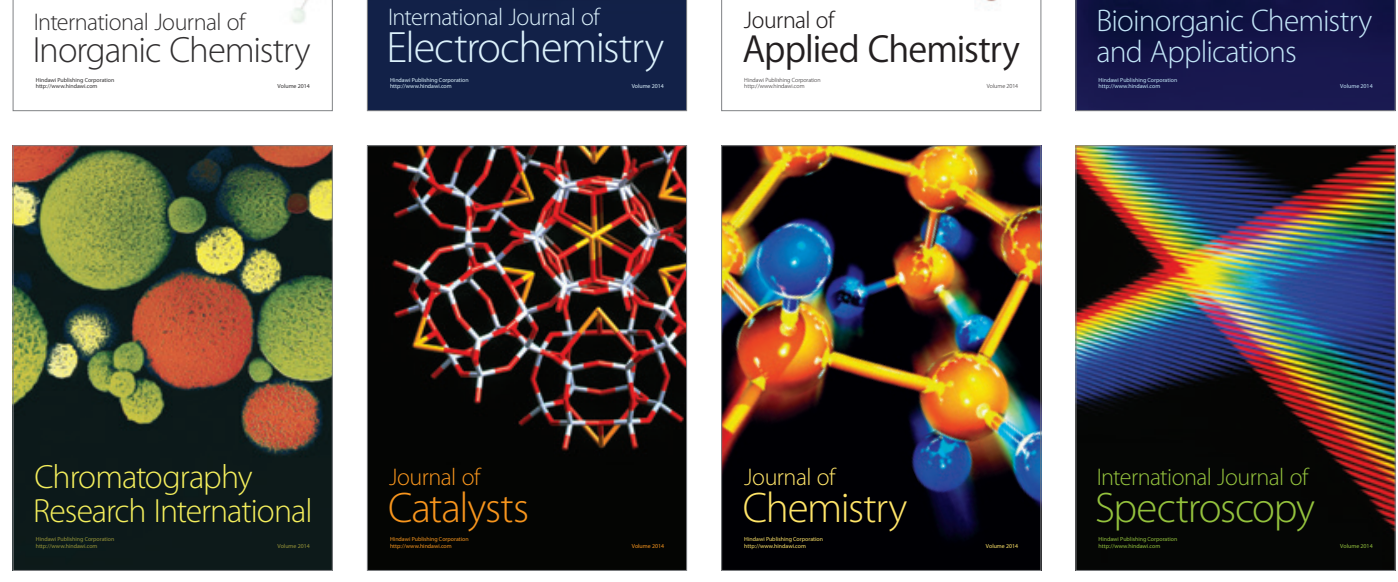